\title{
The flow state analysis of water confined in slit pore by molecular dynamics simulations
}

\author{
Yao Wang ${ }^{1, ~ a, ~ L i q i n ~ H u a n g ~}{ }^{2, b}$ \\ ${ }^{1}$ Nano-Science \& Technology Research Center, Shanghai University, Shanghai 200444, China \\ ${ }^{2}$ School of Mechanical Engineering, Shanghai Dianji University, Shanghai 201306, China \\ awyaocn@foxmail.com, bhuanglq@sdju.edu.cn
}

\begin{abstract}
Keywords: Confined water, Laminar flow, Boundary layer, Slip, Molecular dynamics simulations. Abstract. Space size plays an important role in the properties of confined water. This study aimed to investigate the flow behavior of water confined in a slit pore. Molecular dynamics simulations were acted to analyze the confined water molecules in slit pore. Pressing the plates to form the confined condition and then moving the two plates to opposite directions, the confined water molecules were made in a directed flowing. The flow velocity of the water molecules was then analyzed at the nanoscale. Two crystal silicon surfaces were used to play as the slit pore model. The interaction strength between Si atom and water oxygen atom is lead to the flow behavior. Simulation results show that for the studied case, there is the laminar flow behavior of confined water and at the molecular level boundary slip condition does hold. Moreover, simulation results demonstrate that water molecules confined in the slit are layered and water molecules partially regularly arranged in every layer.
\end{abstract}

\section{Introduction}

Confining geometries that contain thin water films are common in biology, geology, and engineering. For example, restricted cell in biological system [1,2], nanoporous membrane science [3,4], biomimetic materials [5], and microfluidic technologies [6]. Understanding the geometric and flow properties of confined water is important in the theory and practical application.

The flow behavior of fluid confined in a slit is a complex process in which fluid molecules need to overcome two types of interaction strength (i.e., the interaction strength between the pore wall and the fluid molecules and the interactions among the fluid molecules themselves) under a certain driving force. The difference in the interactions of various fluid molecules flowing through a pore leads to different flow properties. In this study, we confined water molecules in a slit pore formed by two solid $\mathrm{Si}(001)$ plates and study the flow state of confined water molecules.

Traditional efforts to study fluid flow through confined structures have been limited to macro-scale flow. Experimentally, atomic force microscopy (AFM) and Surface force apparatus (SFA) are adopted to study the confined fluid [7,8]. Molecular dynamics (MD) simulation is recognized as an effective method for studying the behaviors of water molecules under nanoconfinements. Various MD simulation techniques have been developed, including the external field non-equilibrium molecular dynamics method, the dual control volume grand canonical molecular dynamics method, and so on. When Liquid molecules are confined in a Nano-sized environment, the liquid-solid interfacial interactions show many interesting properties $[9,10]$. A. Opitz and U. Raviv et al. have revealed that the frictional properties of the liquid-solid system are strongly influenced by the thickness of the confined water film [11,12]. L.L. Huang et al. have implemented a series of MD simulations at equilibrium conditions to evaluate the factors affecting the behavior of confined water molecules, including pore plate modification by size, and temperature, and so on, and have demonstrated that the effects of pore size at the nanoscale are more dominant than external effects, such as temperature and pressure [13].

Many studies have been done to investigate the flow properties of confined water. For instance, Y.D. Zhu et al. used the different interaction strength to investigate the effect of pore wall interfacial properties on the flow behavior of water confined in a slit pore, and they have revealed that friction increases with the hydrophilicity of pore walls and further hampers the flow of water under the studied 
confinement conditions [14]. X.X. Qiao et al. used nanocell as an effective method to investigate the nanorheology of confined liquids and the interfacial boundary slip, and they have concluded that the responses of quartz crystal microbalance with the nanocell assembled are dependent on the nanocell confinement thickness, the acoustic impedance of the nanocell lid (parallel plate), as well as the boundary slip on the interface [15]. Yong et al. have used molecular dynamics to model a liquid argon slab confined between two smooth rigid copper walls with an applied velocity at the upper wall to generate a planar Couette flow, and they have the results that liquid structures are strongly correlated with the liquid velocity and density profiles in the flow, and the liquid structures also determine the boundary conditions from pure slip to multilayer locking at liquid-solid interfaces [16]. B. Kim et al. present accurate measurements of shear stress in the bound hydration water layer using noncontact dynamic force microscopy [17].

In this study the molecular dynamics (MD) simulations is performed on confined water molecules by moving the two plates to opposite directions to simulate the flow of confined water. The aim is to investigate the flow behavior of confined water.

\section{Simulation model and method}

The simulation model is show in Fig.1. Using two parallel solid $\mathrm{Si}(001)$ plates to confine water. The Si plates were infinite in the $\mathrm{X}$ and $\mathrm{Y}$ directions. Three-dimensional periodic boundary conditions were applied on the entire simulation system except for the confined water molecules in the Z-direction. The system is composed of $\mathrm{N}=348$ water molecules which are immersed in two parallel Si plates. The total number of silicon atoms in each plate was 384 . The initial slit width is $22 \AA$. The TIP4P model of water was employed [18]. The interaction potentials between the silicon atoms are Stillinger-Weber-like potential [19]. The total interaction potentials between the water molecules and the silicon atoms of the plate surfaces were calculated using the Lennard-Jones potential method [20].

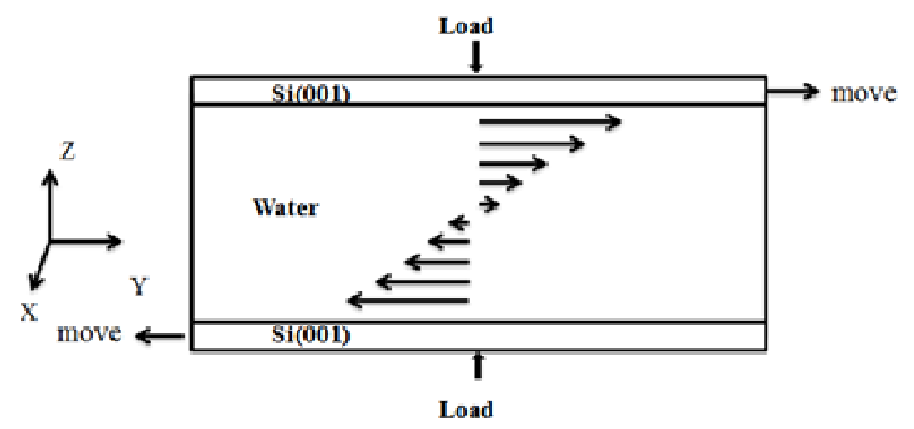

Fig. 1 The physical model of confined water fluid

The procedure is as follows: (1) slit were composed by two Si plates $(2.17 \mathrm{~nm} \times 2.17 \mathrm{~nm} \times 1.62 \mathrm{~nm})$ and placed along the $\mathrm{X}$ and $\mathrm{Y}$ directions. The slit was full by water film $(2.17 \mathrm{~nm} \times 2.17 \mathrm{~nm} \times 2.17 \mathrm{~nm})$ with a density of $1.0 \mathrm{~g} / \mathrm{cm}^{3}$. The system was maintained at a constant temperature $(293 \mathrm{~K})$. After the energy minimization and 2ns MD was run with an integral step of $1.0 \mathrm{fs}$ to obtain the equilibrium configuration. (2) Si plates are loaded $60 \mathrm{nN}$ and $10 \mathrm{~ns}$ MD was run with an integral step of 1.0fs to the final state and now the pore width is about $1.2 \mathrm{~nm}$. (3) To study the flow properties of the confined water, keep the load $60 \mathrm{nN}$, the top and bottom plates were made to slide in opposite directions along the $\mathrm{Y}$-axis at a sliding velocity of $\mathrm{U}_{0}=100 \mathrm{~m} / \mathrm{s}$. MD simulations were performed to run $10 \mathrm{~ns}$ and the last 1 ns trajectory was used for analysis.

During the simulations in this study, the system was maintained at a constant temperature (293 K) by the Gauss-Hoover method. The equations of the motion of water molecules were solved using the Gear-Prediction algorithm with a time step of $1 \mathrm{fs}$. 


\section{Results and discussion}

Spatial distribution of water molecules. In this confined situation, water molecules will form new distribution and the distribution can be described as the density profile of their oxygen atoms along the $Z$-direction and the atoms position profile. The density is defined as the ratio of the local density at position $\mathrm{Z}$ along the slit pore width to the bulk density and is averaged over rectangular shells $(\Delta \mathrm{Z}=$ $0.10 \AA$ ) parallel to the slit wall in the Z-direction. The density is shown in Fig. 2, which clearly shows the water spatial distribution when the water molecules flow in external pressure slit.

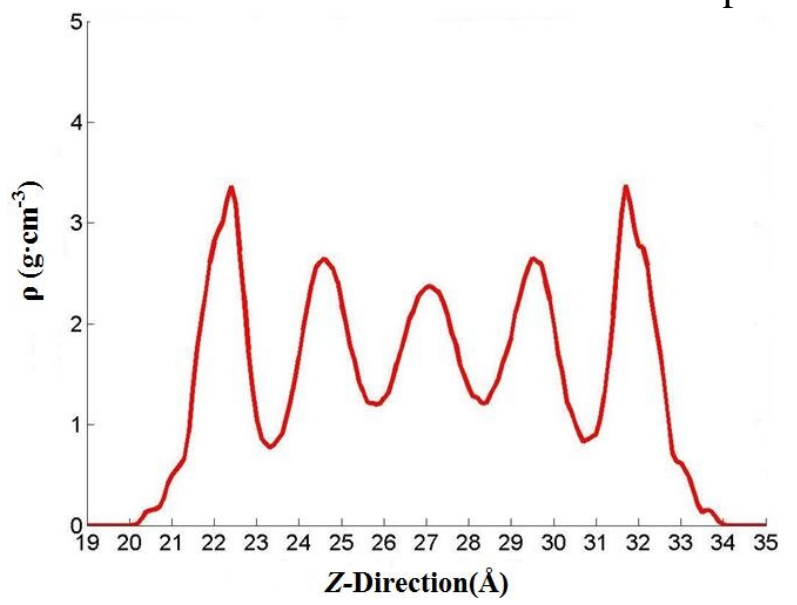

Fig. 2 Density profile for along the Z-direction. The Y-axis represents the density profile of confined water along the Z-direction.

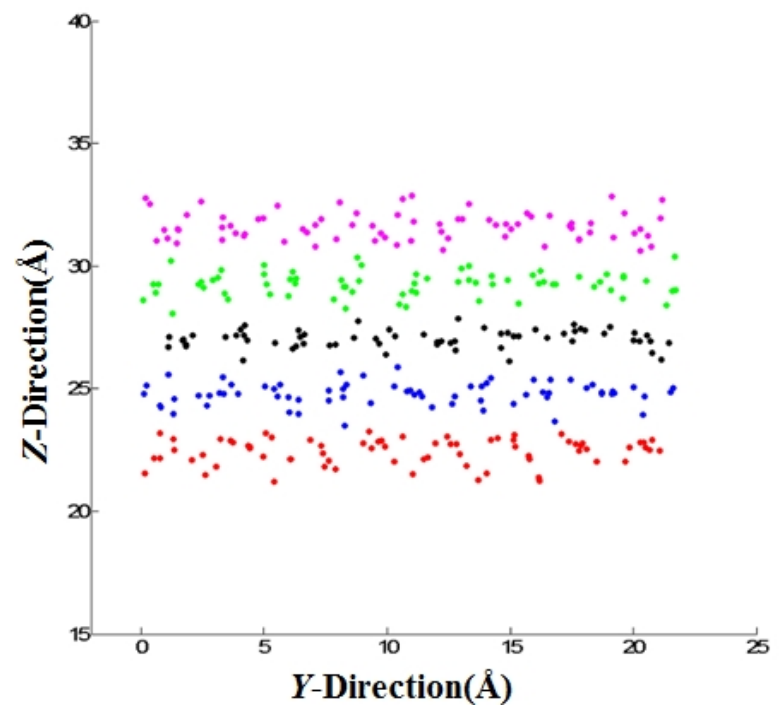

Fig. 3 Confined water molecules position profile along the Z-direction. The Y-axis represents the position of confined water molecules in Y-direction. The bottom layer is the first layer.

For this case, the five obvious peaks of the density are distributed along the Z-direction, suggesting the confined water is laminated flowing. The two first maxima of molecules profile appear near both sides of the plate surfaces, and the three maxima appear in the central region of the slit. For the five peaks, the values of the density turn to 3 , and it indicates that water molecules are layered.

The atoms position profile is shown in Fig. 3. From the Fig. 3, five water layers can be clearly seen. The Fig. 3 also shows the water molecules are layered. The arrangement of water molecules in every layer is also analyzed. As shown in Fig. 4, 4(a) is the arrangement of water molecules in first layer, and the 4(b) is the arrangement of water molecules in the second layer, and so on. From the Fig. 4, the conclusion that water molecules in every layer partially regularly arranged can be got. 

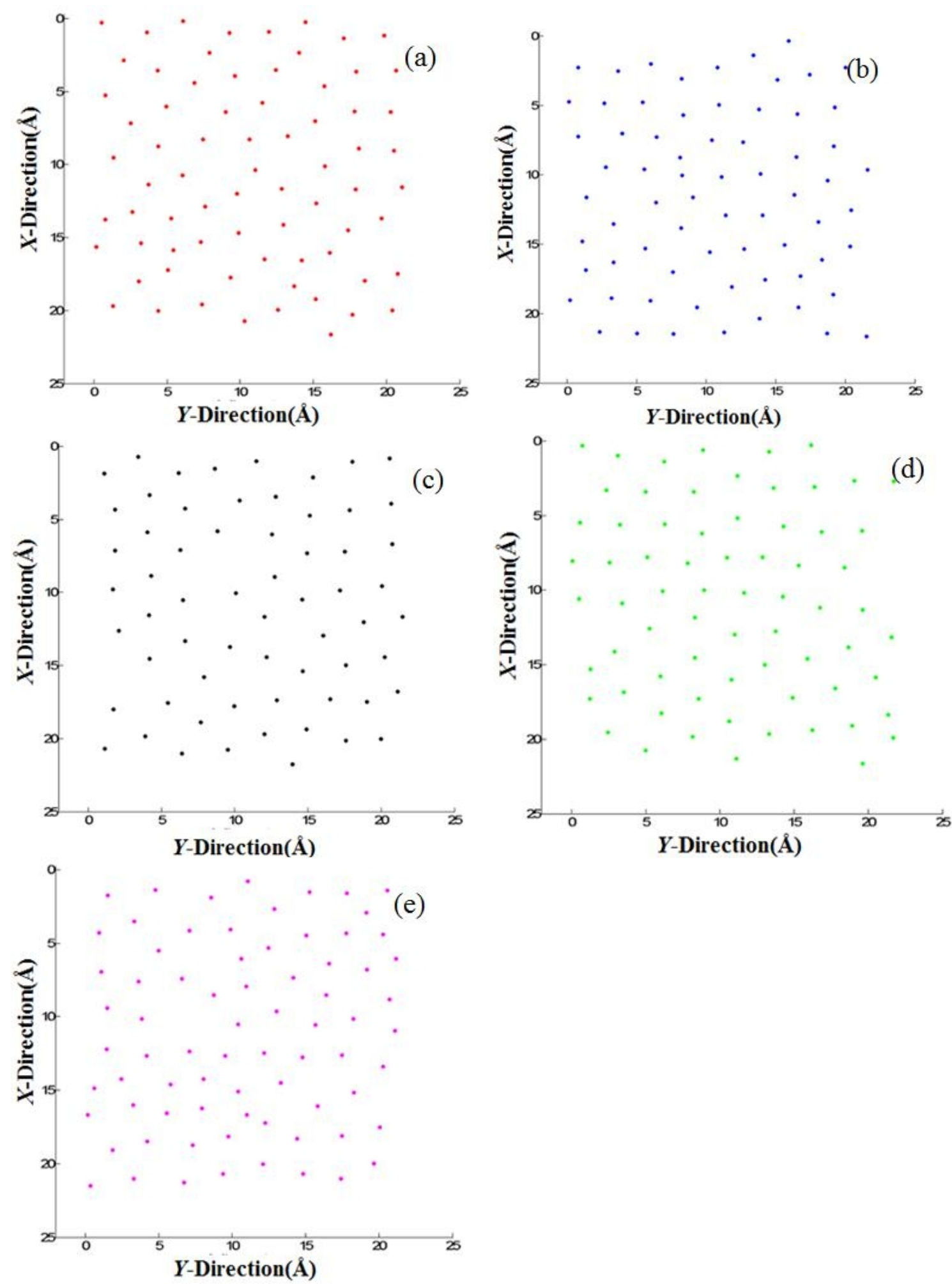

Fig. 4 Arrangement of Confined water molecules in every layer. (a) is the first layer, (b) is the second layer, (c) is the third layer, (d) is the fourth layer, (e) is the fifth layer.

Velocity profiles. The velocity profile along the Z-direction is shown in Fig. 5. For this case, the velocity $\mathrm{U}^{*}$ of confined water increases as the distance from the interface decreases. For the velocity of water molecules near both sides of the wall surfaces is larger, and the water molecules confined in the central region of the slit, $\mathrm{U}^{*}$ is small. The movement of the water molecules is driven by the plates, because the plates keep the same velocity throughout the simulation process. The relative motions of the water molecule is studied, the velocity of the water molecules in the central region is smaller than that of the water molecules near the interfaces. It is to say that the relative motions of water molecules 
in the central region were larger than the water molecules near the sides. So if the pore walls were fixed, and when the confined water flow through the slit, the velocity of water molecules in the central region would faster relative to that of the water molecules near the interfaces and the confined water in this case is laminar flow.

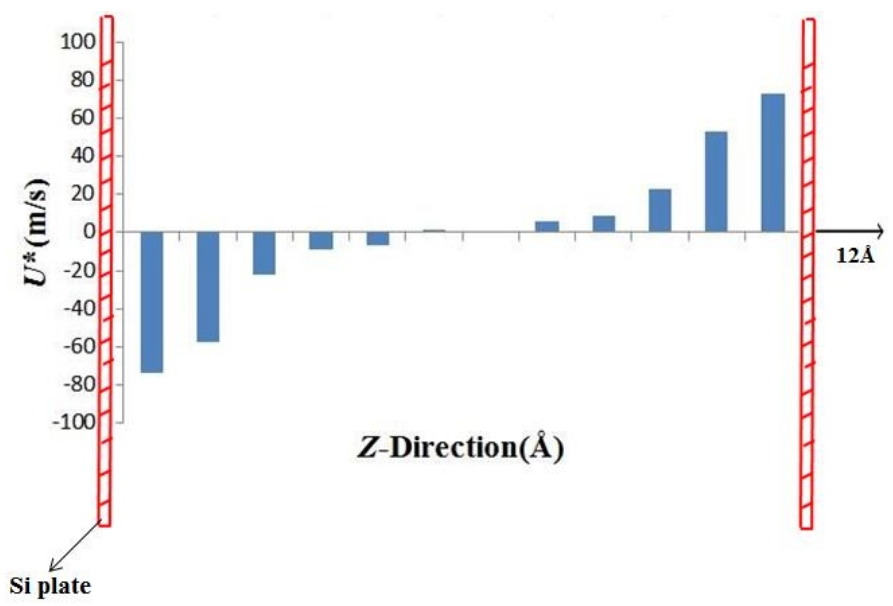

Fig. 5 Confined water velocity profile along the Z-direction. The $\mathrm{Y}$-axis ( $\mathrm{U}^{*}$ ) represents the velocity of confined water in Y-direction.

The relative velocity of confined water to the plates' surfaces is also concerned. At the macroscale no-slip boundary condition usually is adopted when flow properties of a fluid are analyzed. But at the nanoscale, the situation is not the same. As showed in Fig. 5, revealing that the velocity of water molecules near the interfaces is different from that of the plates. Water slip phenomena near the interface are observed suggesting that slip boundary condition arises at the nanoscale. And from the Fig. 5 , the laminar flow behavior of confined water has also been observed .The similar results have also been got in other studies $([14,15])$.

\section{Conclusions}

In this study, we have used MD simulations on confined water molecules between two crystal silicon plates to study the flow behavior of confined fluids. We have analyzed the flow velocity, the spatial distribution of the confined water molecules, and the arrangement of water molecules and concerned the boundary slip. At the molecule level, the flow velocity suggests that the confined water in this case is laminar flow and exit the boundary slip condition. Spatial distribution and the atoms position profile show that the water molecules confined in the slit are layered and confined water partially regularly arranged in every layer.

\section{References}

[1] M.-C. Bellissent-Funel: J. Mol. Liq. Vol. 78 (1998).p. 19

[2] M.-C. Bellissent-Funel: The European Physical Journal E Vol.12 (2003), p. 83

[3] S.F. Zhao, L. Zou, C.Y.Y. Tang: J. Mater. Chem. Vol.396 (2012), p. 1

[4] D. Li, H.T. Wang: J. Mater. Chem. Vol.20 (2010), p. 4551

[5] R. Garcia-Fandino, M.S.P. Sansom: Proc. Natl. Acad. Sci. U.S.A. Vol.109 (2012), p. 6939

[6] H.G. Craighead: Science Vol.290 (2000), p. 1532

[7] R. An, Q.M. Yu, L.Z. Zhang, Y.D. Zhu, X.J. Guo, S.Q. Fu, L.C. Li, C.S. Wang, X.M. Wu, C. Liu, X.H. Lu: Langmuir Vol.28 (2012), p. 15270

[8] S.H. Liu, J.B. Luo, G. Li, C.H. Zhang, X.C. Lu: Appl. Surf. Sci. Vol.254 (2008), p. 7137

[9] W. Sparreboom, A. Vandenberg, J. C. T. Eijkel: Nat. Nanotechnol. Vol.4 (2009), p. 713 
[10] L. Bocquet, E. Charlaix: Chem. Soc. Rev. Vol.39 (2010), p. 1073

[11] A. Opitz, S. I.-U. Ahmed, J. A. Schaefer: Surf. Sci. Vol.504 (2002), p. 199

[12] U. Raviv, S. Giasson, J. Frey, J. Klein: J. Phys. Vol.14 (2012), p. 9275

[13] L.L. Huang, Q. Shao, L.H. Lu, X.H. Lu, L.Z. Zhang, J. Wang, S.Y. Jiang: Phys. Chem. Chem. Phys. Vol.8 (2006), p. 3836

[14] Y.D. Zhu, L.Z. Zhang, X.H. Lu, L.H. Lu, X.M. Wu: Fluid Phase Equilibria Vol.362 (2014), p. 235

[15] X.X. Qiao, X.J. Zhang: Phys. Chem. Chem. Phys. Vol.17 (2015), p. 7224

[16] X. Yong, L.T. Zhang: Physical Review E Vol.82 (2010), p. 056313

[17] B. Kim, S. Kwon, M. Lee, Q. Kim, S. An, W. Jhe: Phys. Rev. Lett. Vol.111 (2013), p. 246102

[18] W.L. Jorgensen, J. Chandrasekhar: J. Chem. Phys. Vol.79 (1983), p. 926

[19] T. Watanabe, H. Fujiwara: Japanese Journal of Applied Physics Vol.38 (1999), p. 366

[20] C.Y. Tang, L.C. Zhang: Nanotechnology Vol.16 (2004), p. 15 